\title{
Existence Theory for Single Positive Solution to Fourth-Order Boundary Value Problems
}

\author{
Ying He \\ School of Mathematics and Statistics, Northeast Petroleum University, Daqing, China \\ Email: heying65338406@163.com
}

Received 25 June 2014; revised 25 July 2014; accepted 5 August 2014

Copyright (C) 2014 by author and Scientific Research Publishing Inc.

This work is licensed under the Creative Commons Attribution International License (CC BY).

http://creativecommons.org/licenses/by/4.0/

(c) (i) Open Access

\begin{abstract}
By fixed point theorem of a mixed monotone operator, we study boundary value problems to nonlinear singular fourth-order differential equations, and provide sufficient conditions for the existence and uniqueness of positive solution. The nonlinear term in the differential equation may be singular.
\end{abstract}

\section{Keywords}

Mixed Monotone Operator, Singular, Existence, Uniqueness

\section{Introduction}

Fourth-order differential equations play an important role in various fields of science and engineering. With the help of boundary value conditions, we can describe the natural phenomena and mathematical model more accurately. Therefore, the fourth-order differential equations have received much attention and the theory and application have been greatly developed (see [1]-[4] and their references). Most of the results told us that the equations had at least single and multiple positive solutions. In papers [1]-[3], the authors obtained some newest results for the singular fourth-order boundary value problems. But there is no result on the uniqueness of solution in them.

In this paper, we consider the following singular fourth-order boundary value problem:

$$
\left\{\begin{array}{l}
{\left[p(t) x^{\prime \prime \prime}(t)\right]^{\prime}+q(t) x^{\prime \prime}(t)=\lambda f(t, x(t)), \quad 0<t<1, \lambda>0} \\
\alpha_{1} x(0)-\beta_{1} x^{\prime}(0)=0 \\
\gamma_{1} x(1)+\delta_{1} x^{\prime}(1)=0 \\
\alpha_{2} x^{\prime \prime}(0)-\beta_{2} x^{\prime \prime \prime}(0)=0 \\
\gamma_{2} x^{\prime \prime}(1)+\delta_{2} x^{\prime \prime \prime}(1)=0
\end{array}\right.
$$


Throughout this paper, we always suppose that

$\left(S_{1}\right) \quad p(t) \in C^{1}([0,1], R), p(t)>0, q(t) \in C([0,1], R), q(t) \leq 0, \alpha_{i}, \beta_{i}, \gamma_{i}, \delta_{i} \geq 0, \quad(i=1,2)$,

and $\beta_{i} \gamma_{i}+\alpha_{i} \gamma_{i}+\alpha_{i} \delta_{i}>0(i=1,2) . f \in C((0,1) \times(0,+\infty),(0,+\infty))$.

Moreover, $f(t, u)$ may be singular at $t=0, t=1$, or $x=0$.

Equation (1.1) is often referred to as the deformation for an elastic beam under a variety of boundary conditions. A brief discussion of the physical interpretation under some boundary conditions associated with the linear beam equation can be found in Zill and Cullen [5]. In this article, we consider the existence and uniqueness of positive solutions for fourth-order singular boundary value problems by using mixed monotone method.

\section{Preliminary}

Let $P$ be a normal cone of a Banach space $E$, and $e \in P$ with $\|e\| \leq 1, e \neq \theta$. Define

$$
Q_{e}=\{x \in P \mid x \neq \theta \text {, there exist constants } m, M>0 \text { such that } m e \leq x \leq M e\} .
$$

Now we give a definition(see [7]).

Definition 2.1. Assume $A: Q_{e} \times Q_{e} \rightarrow Q_{e}$. $A$ is said to be mixed monotone if $A(x, y)$ is nondecreasing in $x$ and nonincreasing in $y$, i.e. if $x_{1} \leq x_{2}\left(x_{1}, x_{2} \in Q_{e}\right)$ implies $A\left(x_{1}, y\right) \leq A\left(x_{2}, y\right)$ for any $y \in Q_{e}$, and $y_{1} \leq y_{2}\left(y_{1}, y_{2} \in Q_{e}\right)$ implies $A\left(x, y_{1}\right) \geq A\left(x, y_{2}\right)$ for any $x \in Q_{e} \cdot x^{*} \in Q_{e}$ is said to be a fixed point of $A$ if $A\left(x^{*}, x^{*}\right)=x^{*}$.

Theorem 2.1. Suppose that $A: Q_{e} \times Q_{e} \rightarrow Q_{e}$ is a mixed monotone operator and $\exists$ a constant $\alpha, 0 \leq \alpha<1$, such that

$$
A\left(t x, \frac{1}{t} y\right) \geq t^{\alpha} A(x, y) \quad \forall x, y \in Q_{e}, 0<t<1 .
$$

Then $A$ has a unique fixed point $\quad x^{*} \in Q_{e}$. Moreover, for any $\left(x_{0}, y_{0}\right) \in Q_{e} \times Q_{e}$

satisfy

$$
x_{n}=A\left(x_{n-1}, y_{n-1}\right), \quad y_{n}=A\left(y_{n-1}, x_{n-1}\right), \quad n=1,2, \cdots,
$$

where

$$
x_{n} \rightarrow x^{*}, \quad y_{n} \rightarrow x^{*}
$$

$$
\left\|x_{n}-x^{*}\right\|=o\left(1-r^{\alpha^{n}}\right), \quad\left\|y_{n}-y^{*}\right\|=o\left(1-r^{\alpha^{n}}\right),
$$

$0<r<1, r$ is a constant from $\left(x_{0}, y_{0}\right)$.

Theorem 2.2. (See [7]): Suppose that $A: Q_{e} \times Q_{e} \rightarrow Q_{e}$ is a mixed monotone operator and $\exists$ a constant $\alpha \in(0,1)$ such that (2.1) holds. If $x_{\lambda}^{*}$ is a unique solution of equation

$$
A(x, x)=\lambda x, \quad \lambda>0
$$

in $Q_{e}$, then $\left\|x_{\lambda}^{*}-x_{\lambda_{0}}^{*}\right\| \rightarrow 0, \lambda \rightarrow \lambda_{0}$. If $0<\alpha<\frac{1}{2}$, then $0<\lambda_{1}<\lambda_{2}$ implies $x_{\lambda_{1}}^{*} \geq x_{\lambda_{2}}^{*}, x_{\lambda_{1}}^{*} \neq x_{\lambda_{2}}^{*}$, and

$$
\lim _{\lambda \rightarrow+\infty}\left\|x_{\lambda}^{*}\right\|=0, \quad \lim _{\lambda \rightarrow 0^{+}}\left\|x_{\lambda}^{*}\right\|=+\infty .
$$

\section{Uniqueness Positive Solution of Problem (1.1)}

This section discusses the problem

$$
\left\{\begin{array}{l}
{\left[p(t) x^{\prime \prime \prime}(t)\right]^{\prime}+q(t) x^{\prime \prime}(t)=\lambda f(t, x(t)), \quad 0<t<1, \lambda>0} \\
\alpha_{1} x(0)-\beta_{1} x^{\prime}(0)=0 \\
\gamma_{1} x(1)+\delta_{1} x^{\prime}(1)=0 \\
\alpha_{2} x^{\prime \prime}(0)-\beta_{2} x^{\prime \prime \prime}(0)=0 \\
\gamma_{2} x^{\prime \prime}(1)+\delta_{2} x^{\prime \prime \prime}(1)=0
\end{array}\right.
$$


Throughout this section, we assume that

$$
f(t, x)=z(t)(g(x)+h(x)), \quad t \in(0,1),
$$

where

$$
\begin{aligned}
& g:[0,+\infty) \rightarrow[0,+\infty) \text { is continuous and nondecreasing; } \\
& h:(0,+\infty) \rightarrow(0,+\infty) \text { is continuous and nonincreasing. }
\end{aligned}
$$

Let $Q=I \times I$ and $Q_{1}=\{(t, s) \in Q \mid 0 \leq t \leq s \leq 1\}, Q_{2}=\{(t, s) \in Q \mid 0 \leq s \leq t \leq 1\}$. We denote the Green's functions for the following boundary value problems

and

$$
\left\{\begin{array}{l}
-x^{\prime \prime}(t)=0, \quad 0<t<1 \\
\alpha_{1} x(0)-\beta_{1} x^{\prime}(0)=0 \\
\gamma_{1} x(1)+\delta_{1} x^{\prime}(1)=0
\end{array}\right.
$$

$$
\left\{\begin{array}{l}
-\left(p(t) x^{\prime}(t)\right)^{\prime}-q(t) x(t)=0, \quad 0<t<1, \\
\alpha_{2} x(0)-\beta_{2} x^{\prime}(0)=0 \\
\alpha_{2} x(1)+\beta_{2} x^{\prime}(1)=0,
\end{array}\right.
$$

by $H(t, s)$ and $G(t, s)$, respectively. It is well known that $H(t, s)$ and $G(t, s)$ can be written by

$$
H(x, y):=\frac{1}{\rho_{1}} \begin{cases}\left(\beta_{1}+\alpha_{1} t\right)\left(\delta_{1}+\gamma_{1}(1-s)\right), & (t, s) \in Q_{1}, \\ \left(\beta_{1}+\alpha_{1} s\right)\left(\delta_{1}+\gamma_{1}(1-t)\right), & (t, s) \in Q_{2},\end{cases}
$$

where $\rho_{1}=\beta_{1} \gamma_{1}+\alpha_{1} \gamma_{1}+\alpha_{1} \delta_{1}>0$ and

$$
G(t, s):=\frac{1}{\omega} \begin{cases}m(t) n(s), & (t, s) \in Q_{1}, \\ m(s) n(t), & (t, s) \in Q_{2} .\end{cases}
$$

Lemma 3.1. Suppose that $\left(S_{1}\right)$ holds, then the Green's function $G(t, s)$, possesses the following properties:

1) $m(t) \in C^{2}(I, R)$ is increasing and $m(t)>0, x \in(0,1]$.

2) $n(t) \in C^{2}(I, R)$ is decreasing and $n(t)>0, x \in[0,1)$.

3) $(\mathrm{Lm})(t) \equiv 0, m(0)=\beta_{2}, m^{\prime}(0)=\alpha_{2}$.

4) $(L n)(t) \equiv 0, n(1)=\delta_{2}, n^{\prime}(1)=-\gamma_{2}$.

5) $\omega$ is a positive constant. Moreover, $p(t)\left(m^{\prime}(t) n(t)-m(t) n^{\prime}(t)\right) \equiv \omega$.

6) $G(t, s)$ is continuous and symmetrical over $Q$.

7) $G(t, s)$ has continuously partial derivative over $Q_{1}, Q_{2}$.

8) For each fixed $s \in I, G(t, s)$ satisfies $L G(t, s)=0$ for $s \neq t, t \in I$. Moreover, $R_{1}(G)=R_{2}(G)=0$ for $s \in(0,1)$.

9) $G_{t}^{\prime}$ has discontinuous point of the first kind at $t=s$ and

$$
G_{t}^{\prime}(s+0, s)-G_{t}^{\prime}(s-0, s)=-\frac{1}{p(s)}, \quad s \in(0,1)
$$

Following from Lemma 3.1, it is easy to see that

(a) $H(t, s) \leq H(t, t), \quad H(t, s) \leq H(s, s)$,

$$
H(t, s) \geq \frac{\rho_{1}}{\left(\alpha_{1}+\beta_{1}\right)\left(\delta_{1}+\gamma_{1}\right)} H(t, t) H(s, s) \text {, for }(t, s) \in[0,1] \times[0,1] .
$$

(b) $G(t, s) \leq G(t, t)=\frac{1}{\omega} m(t) n(t), \quad G(t, s) \leq G(s, s)$,

$$
G(t, s) \geq G(t, t) G(s, s) \frac{w}{m(1) n(0)} \text {, for }(t, s) \in[0,1] \times[0,1] .
$$


Suppose that $x$ is a positive solution of (1.1). Then

$$
x(t)=\lambda \int_{0}^{1} \int_{0}^{1} H(t, \tau) G(\tau, s) f(s, x(s)) \mathrm{d} s \mathrm{~d} \tau \quad 0 \leq t \leq 1 .
$$

By using (3.3) and (a), we see that for every positive solution $x$ one has

$$
\begin{aligned}
& \|x\| \leq \lambda \int_{0}^{1} \int_{0}^{1} H(\tau, \tau) G(\tau, s) f(s, x(s)) \mathrm{d} s \mathrm{~d} \tau, \\
& x(t) \geq \frac{\rho_{1}}{\left(\alpha_{1}+\beta_{1}\right)\left(\delta_{1}+\gamma_{1}\right)} H(t, t) \lambda \int_{0}^{1} \int_{0}^{1} H(\tau, \tau) G(\tau, s) f(s, x(s)) \mathrm{d} s \mathrm{~d} \tau \geq \frac{\rho_{1}}{\left(\alpha_{1}+\beta_{1}\right)\left(\delta_{1}+\gamma_{1}\right)}\|x\|
\end{aligned}
$$

where $\|x\|=\sup \{|x(t)| ; 0 \leq t \leq 1\}$. Let

$$
K(t, s)=\int_{0}^{1} H(t, \tau) G(\tau, s) \mathrm{d} \tau .
$$

Thus by (3.3) one has

$$
x(t)=\lambda \int_{0}^{1} K(t, s) f(s, x(s)) \mathrm{d} s \mathrm{~d} \tau, \quad 0 \leq t \leq 1
$$

by (a) one has

$$
\frac{\rho_{1}}{\left(\alpha_{1}+\beta_{1}\right)\left(\delta_{1}+\gamma_{1}\right)} H(t, t) \int_{0}^{1} H(\tau, \tau) G(\tau, s) \mathrm{d} \tau \leq K(t, s) \leq H(t, t) \int_{0}^{1} G(\tau, s) \mathrm{d} \tau .
$$

Let $P=\{x \in C[0,1] \mid x(t) \geq 0, \forall t \in[0,1]\}$. Obviously, $P$ is a normal cone of Banach space $C[0,1]$.

Theorem 3.1. Suppose that there exists $\alpha \in(0,1)$ such that

$$
\begin{aligned}
& g(t x) \geq t^{\alpha} g(x) \\
& h\left(t^{-1} x\right) \geq t^{\alpha} h(x)
\end{aligned}
$$

for any $t \in(0,1)$ and $x>0$, and $z \in C((0,1),(0, \infty))$ satisfies

$$
\int_{0}^{1} H^{-\alpha}(s, s) z(s) \mathrm{d} s<+\infty .
$$

Then (1.1) has a unique positive solution $x_{\lambda}^{*}(t)$. And moreover, $0<\lambda_{1}<\lambda_{2}$ implies $x_{\lambda_{1}}^{*} \leq x_{\lambda_{2}}^{*}, x_{\lambda_{1}}^{*} \neq x_{\lambda_{2}}^{*}$. If $\alpha \in\left(0, \frac{1}{2}\right)$, then

$$
\lim _{\lambda \rightarrow 0^{+}}\left\|x_{\lambda}^{*}\right\|=0, \quad \lim _{\lambda \rightarrow+\infty}\left\|x_{\lambda}^{*}\right\|=+\infty .
$$

Proof. Since (3.5) holds, let $t^{-1} x=y$, one has

$$
h(y) \geq t^{\alpha} h(t y)
$$

then

$$
h(t y) \leq \frac{1}{t^{\alpha}} h(y), \forall t \in(0,1), y>0 .
$$

Let $y=1$. The above inequality is

$$
h(t) \leq \frac{1}{t^{\alpha}} h(1), \forall t \in(0,1) .
$$

From (3.5), (3.7) and (3.8), one has

$$
\begin{aligned}
& h\left(t^{-1} x\right) \geq t^{\alpha} h(x), \quad h\left(\frac{1}{t}\right) \geq t^{\alpha} h(1), \\
& h(t x) \leq \frac{1}{t^{\alpha}} h(x), \quad h(t) \leq \frac{1}{t^{\alpha}} h(1), t \in(0,1), \quad x>0 .
\end{aligned}
$$


Similarly, from (3.5), one has

$$
g(t x) \geq t^{\alpha} g(x), \quad g(t) \geq t^{\alpha} g(1), \quad t \in(0,1), \quad x>0 .
$$

Let $t=\frac{1}{x}, x>1$, one has

$$
g(x) \leq x^{\alpha} g(1), \quad x \geq 1 .
$$

Let $e(t)=\frac{\rho_{1}}{\left(\alpha_{1}+\beta_{1}\right)\left(\delta_{1}+\gamma_{1}\right)} H(t, t)$. It is clear that $\|e\|<1$, and now let

$$
Q_{e}=\left\{x \in C[0,1] \mid \frac{1}{M} e(t) \leq x(t) \leq M e(t), t \in[0,1]\right\}
$$

where $M>1$ is chosen such that

$$
\begin{aligned}
M> & \max \left\{\left\{\lambda g(1)\left(\frac{\rho_{1}}{\left(\alpha_{1}+\beta_{1}\right)\left(\delta_{1}+\gamma_{1}\right)}\right)^{-1} \int_{0}^{1} \int_{0}^{1} G(\tau, s) z(s) \mathrm{d} s \mathrm{~d} \tau\right.\right. \\
& \left.+\lambda h(1)\left(\frac{\rho_{1}}{\left(\alpha_{1}+\beta_{1}\right)\left(\delta_{1}+\gamma_{1}\right)}\right)^{-\alpha-1} \int_{0}^{1} \int_{0}^{1} G(\tau, s) z(s) H^{-\alpha}(s, s) \mathrm{d} s \mathrm{~d} \tau\right\} \\
& \left\{\lambda g(1)\left(\frac{\rho_{1}}{\left(\alpha_{1}+\beta_{1}\right)\left(\delta_{1}+\gamma_{1}\right)}\right)^{\frac{1}{1-\alpha}} \int_{0}^{1} \int_{0}^{1} H(\tau, \tau) G(\tau, s) z(s) H^{\alpha}(s, s) \mathrm{d} s \mathrm{~d} \tau\right. \\
& \left.\left.+\lambda h(1) \int_{0}^{1} \int_{0}^{1} H(\tau, \tau) G(\tau, s) z(s) \mathrm{d} \tau \mathrm{d} s\right\}^{-\frac{1}{1-\alpha}}\right\} .
\end{aligned}
$$

For any $x, y \in Q_{e}$, we define

$$
A_{\lambda}(x, y)(t)=\lambda \int_{0}^{1} K(t, s) z(s)[g(x(s))+h(y(s))] \mathrm{d} s \quad \forall t \in[0,1] .
$$

First we show that $A_{\lambda}: Q_{e} \times Q_{e} \rightarrow Q_{e}$. Let $x, y \in Q_{e}$, from (3.10) and (3.11) we have

$$
g(x(t)) \leq g(\operatorname{Me}(t)) \leq g(M) \leq M^{\alpha} g(1)
$$

and from (3.9) we have

$$
h(y(t)) \leq h\left(\frac{1}{M} e(t)\right) \leq M^{\alpha} e^{-\alpha}(t) h(1) .
$$

Then from (3.4) and (3.13) we have

$$
\begin{aligned}
& A_{\lambda}(x, y)(t)=\lambda \int_{0}^{1} H(t, \tau) G(\tau, s) z(s)[g(x(s))+h(y(s))] \mathrm{d} s \mathrm{~d} \tau \\
& \leq \lambda H(t, t)\left\{\int_{0}^{1} \int_{0}^{1} G(\tau, s) z(s) g(x(s)) \mathrm{d} s \mathrm{~d} \tau+\int_{0}^{1} \int_{0}^{1} G(\tau, s) z(s) h(y(s)) \mathrm{d} s \mathrm{~d} \tau\right\} \\
& \leq \lambda H(t, t)\left\{M^{\alpha} g(1) \int_{0}^{1} \int_{0}^{1} G(\tau, s) z(s) \mathrm{d} s \mathrm{~d} \tau+M^{\alpha} h(1) \int_{0}^{1} \int_{0}^{1} G(\tau, s) z(s) e^{-\alpha}(s) \mathrm{d} s \mathrm{~d} \tau\right\} \\
& =\lambda H(t, t)\left\{M^{\alpha} g(1) \int_{0}^{1} \int_{0}^{1} G(\tau, s) z(s) \mathrm{d} s \mathrm{~d} \tau+M^{\alpha} h(1)\left(\frac{\rho_{1}}{\left(\alpha_{1}+\beta_{1}\right)\left(\delta_{1}+\gamma_{1}\right)}\right)^{-\alpha} \int_{0}^{1} \int_{0}^{1} G(\tau, s) z(s) H^{-\alpha}(s, s) \mathrm{d} s \mathrm{~d} \tau\right\} \\
& \leq e(t) M \quad t \in[0,1] .
\end{aligned}
$$


On the other hand, for any $x, y \in Q_{e}$, from (3.9) and (3.10), we have

$$
\begin{aligned}
& g(x(t)) \geq g\left(\frac{1}{M} e(t)\right) \geq M^{-\alpha} e^{\alpha}(t) g(1), \\
& h(y(t)) \geq h(M e(t)) \geq h(M) \geq M^{-\alpha} h(1) .
\end{aligned}
$$

Thus, from (3.15), we have

$$
\begin{aligned}
& A_{\lambda}(x, y)(t)=\lambda \int_{0}^{1} H(\tau, \tau) G(\tau, s) z(s)[g(x(s))+h(y(s))] \mathrm{d} s \\
& \geq \lambda \frac{\rho_{1}}{\left(\alpha_{1}+\beta_{1}\right)\left(\delta_{1}+\gamma_{1}\right)} H(t, t)\left\{\int_{0}^{1} \int_{0}^{1} H(\tau, \tau) G(\tau, s) z(s) g(x(s)) \mathrm{d} \tau \mathrm{d} s+\int_{0}^{1} \int_{0}^{1} H(\tau, \tau) G(\tau, s) z(s) h(y(s)) \mathrm{d} \tau \mathrm{d} s\right\} \\
& \geq \lambda e(t)\left\{M^{-\alpha} g(1) \int_{0}^{1} \int_{0}^{1} H(\tau, \tau) G(\tau, s) z(s) e^{\alpha}(s) \mathrm{d} \tau \mathrm{d} s+M^{-\alpha} h(1) \int_{0}^{1} \int_{0}^{1} H(\tau, \tau) G(\tau, s) z(s) \mathrm{d} \tau \mathrm{d} s\right\} \\
& =\lambda e(t)\left\{M^{-\alpha} g(1)\left(\frac{\rho_{1}}{\left(\alpha_{1}+\beta_{1}\right)\left(\delta_{1}+\gamma_{1}\right)}\right)^{\alpha} \int_{0}^{1} \int_{0}^{1} H(\tau, \tau) G(\tau, s) H^{\alpha}(s, s) z(s) \mathrm{d} \tau \mathrm{d} s\right. \\
& \left.\quad+M^{-\alpha} h(1) \int_{0}^{1} \int_{0}^{1} H(\tau, \tau) G(\tau, s) z(s) \mathrm{d} \tau \mathrm{d} s\right\} \\
& \geq e(t) \frac{1}{M} .
\end{aligned}
$$

So, $A_{\lambda}$ is well defined and $A_{\lambda}\left(Q_{e} \times Q_{e}\right) \subset Q_{e}$.

Next, for any $l \in(0,1)$, one has

$$
\begin{aligned}
A_{\lambda}\left(l x, l^{-1} y\right)(t) & =\lambda \int_{0}^{1} K(t, s) z(s)\left[g(l x(s))+h\left(l^{-1} y(s)\right)\right] \mathrm{d} s \\
& \geq \lambda \int_{0}^{1} K(t, s) z(s)\left[l^{\alpha} g(x(s))+l^{\alpha} h(y(s))\right] \mathrm{d} s \\
& =l^{\alpha} A_{\lambda}(x, y)(t), \quad t \in[0,1] .
\end{aligned}
$$

So the conditions of Theorems 2.1 and 2.2 hold. Therefore there exists a unique $x_{\lambda}^{*} \in Q_{e}$ such that $A_{\lambda}\left(x^{*}, x^{*}\right)=x_{\lambda}^{*}$. It is easy to check that $x_{\lambda}^{*}$ is a unique positive solution of (1.1) for given $\lambda>0$. Moreover, Theorem 2.2 means that if $0<\lambda_{1}<\lambda_{2}$ then $x_{\lambda_{1}}^{*}(t) \leq x_{\lambda_{2}}^{*}(t), \quad x_{\lambda_{1}}^{*}(t) \neq x_{\lambda_{2}}^{*}(t)$, and if $\alpha \in\left(0, \frac{1}{2}\right)$, then

$$
\lim _{\lambda \rightarrow 0^{+}}\left\|x_{\lambda}^{*}\right\|=0, \quad \lim _{\lambda \rightarrow+\infty}\left\|x_{\lambda}^{*}\right\|=+\infty .
$$

This completes the proof.

Example. Consider the following singular fourth-order boundary value problem:

$$
\left\{\begin{array}{l}
{\left[p(t) x^{\prime \prime \prime}(t)\right]^{\prime}+q(t) x^{\prime \prime}(t)=\lambda\left(\mu x^{a}+x^{-b}\right), \quad 0<t<1,} \\
\alpha_{1} x(0)-\beta_{1} x^{\prime}(0)=0, \\
\gamma_{1} x(1)+\delta_{1} x^{\prime}(1)=0 \\
\alpha_{2} x^{\prime \prime}(0)-\beta_{2} x^{\prime \prime \prime}(0)=0, \\
\gamma_{2} x^{\prime \prime}(1)+\delta_{2} x^{\prime \prime \prime}(1)=0,
\end{array}\right.
$$

$\begin{gathered}\text { where } \\ \text { Let }\end{gathered}, a, b>0, \mu \geq 0, \max \{a, b\}<1$, satisfies $\int_{0}^{1} H^{-\alpha}(s, s) z(s) \mathrm{d} s<+\infty$.

$$
\alpha=\max \{a, b\}, g(x)=\mu x^{a}, h(x)=x^{-b}, z(t)=1 .
$$

Thus $0<\alpha<1$ and for any $t \in(0,1) \quad x>0, y>0$,

$$
g(t x)=t^{a} g(x) \geq t^{\alpha} g(x), \quad h\left(t^{-1} x\right)=t^{b} h(x) \geq t^{\alpha} h(x) .
$$


Now Theorem 3.1 guarantees that the above equation has a positive solution.

\section{Funding}

Project was supported by Heilongjiang Province Education Department Natural Science Research Item, China (12541076).

\section{References}

[1] Jiang, D.Q., Liu, H. and Xu, X. (2005) Nonresonant Singular Fourth-Order Boundary Value Problems. Applied Mathematics Letters, 18, 69-75. http://dx.doi.org/10.1016/j.aml.2003.05.016

[2] Jiang, D.Q. (2000) Multiple Positive Solutions to Singular Boundary Value Problems for Superlinear Higher Order ODEs. Computers Mathematics with Applications, 40, 249-259. http://dx.doi.org/10.1016/S0898-1221(00)00158-9

[3] Liu, Y. (2003) Structure of a Class of Singular Boundary Value Problem with Superlinear Effect. Journal of Mathematical Analysis and Applications, 284, 64-75. http://dx.doi.org/10.1016/S0022-247X(03)00214-2

[4] Kong, L. and Wang, J. (2001) The Green's Function for $(k, n-k)$ Conjugate Boundary Value Problems and Its Applications. Journal of Mathematical Analysis and Applications, 255, 404-422. http://dx.doi.org/10.1006/jmaa.2000.7158

[5] Zill, D.G. and Cullen, M.R. (2001) Differential Equations with Boundary-Value Problems. 5th Edition, Brooks Cole, Belmont.

[6] Liu, Y. (2004) Multiple Positive Solutions of Nonlinear Singular Boundary Value Problem for Fourth Order Equations. Applied Mathematics Letters, 17, 747-757. http://dx.doi.org/10.1016/j.aml.2004.06.001

[7] Guo, D. (2000) The Order Methods in Nonlinear Analysis. Shandong Technology and Science Press, Jinan. 
Scientific Research Publishing (SCIRP) is one of the largest Open Access journal publishers. It is currently publishing more than 200 open access, online, peer-reviewed journals covering a wide range of academic disciplines. SCIRP serves the worldwide academic communities and contributes to the progress and application of science with its publication.

Other selected journals from SCIRP are listed as below. Submit your manuscript to us via either submit@scirp.org or Online Submission Portal.
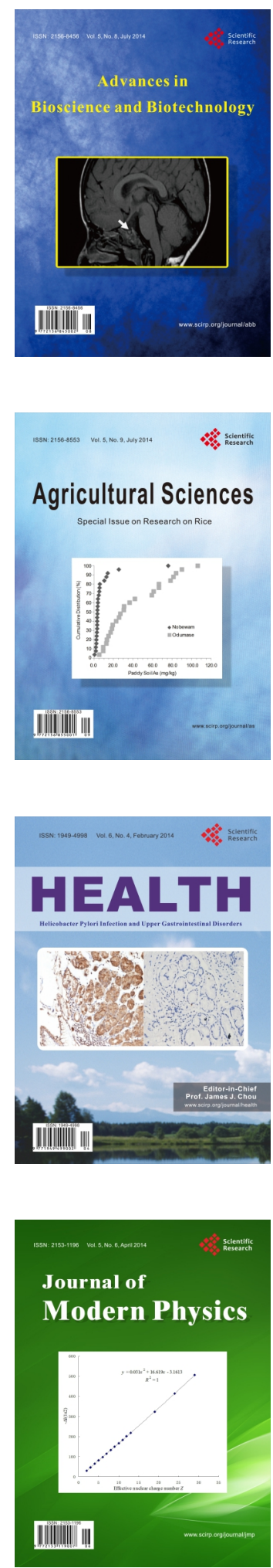
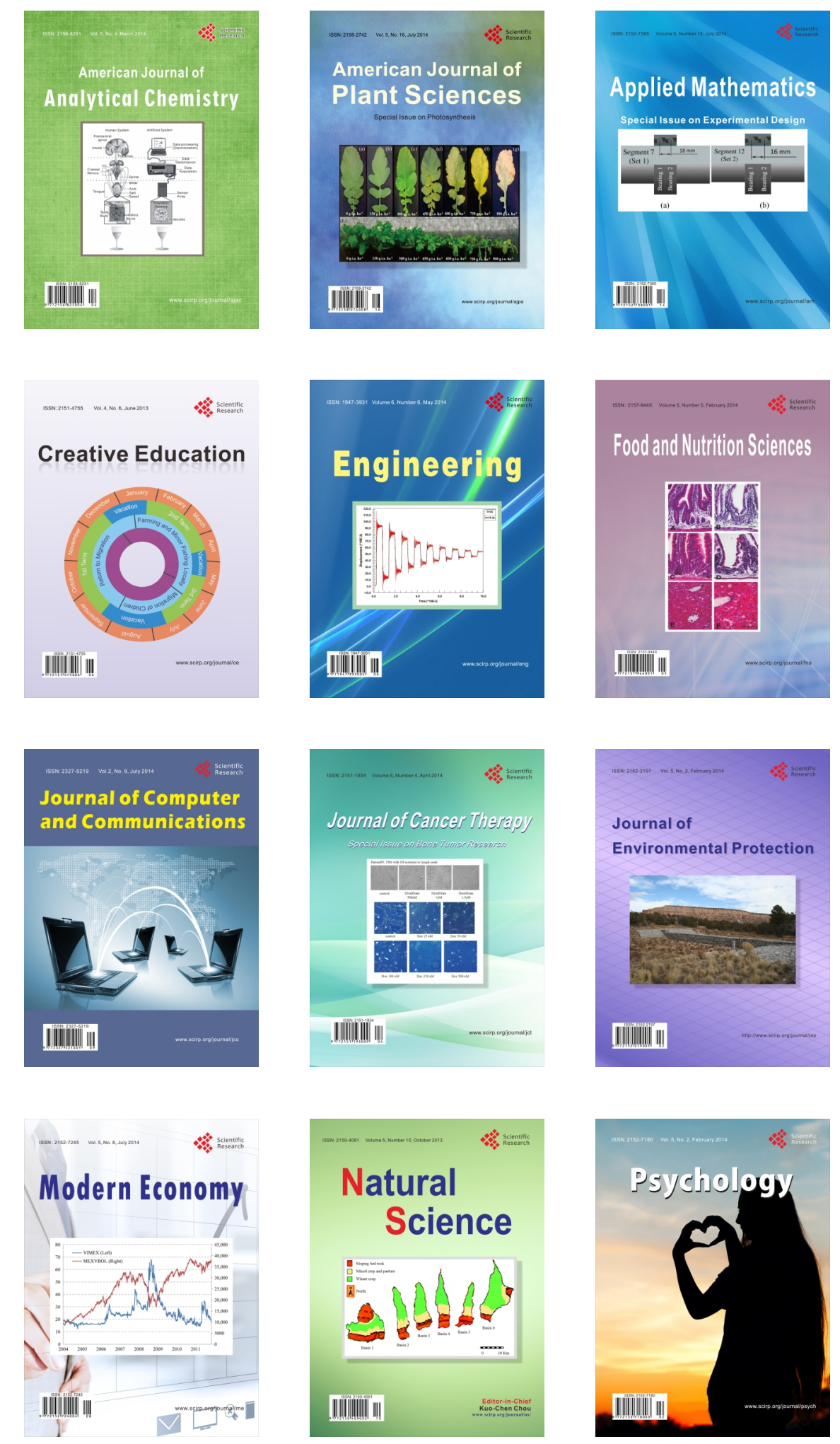